\title{
A Computer-Aided 3-D Geometry of Acute and Chronic Zonal Necrosis: Three-D Tangent Counting Applied in an Attempt to Re-Examine the Structure of the Human Liver
}

\author{
Yasuyuki Nakamura and Tohru Takahashi \\ Department of Pathology, Institute of Development, Aging \\ and Cancer, Tohoku University, Sendai 980-8575
}

\begin{abstract}
Nakamura, Y. and Takahashi, T. A Computer-Aided 3-D Geometry of Acute and Chronic Zonal Necrosis: Three-D Tangents Counting Applied in an Attempt to Re-Examine the Structure of the Human Liver. Tohoku J. Exp. Med., $1998,184(3), 207-227$ - In an attempt to examine the feasibility of the acinar concept in the liver of humans, the spatial distribution of zonal necrosis and its relation with the blood vessels were studied in terms of 3-D tissue microstructure. The material was five autopsy livers, two from patients of acute cresol intoxication and three with long-standing heart failure. Examined were: 1) whether the surviving areas (zones 1,2 ) in acute zonal necrosis have surfaces convex toward the necrotic zone 3 as illustrated in the acinar schema, and 2) whether the necrosis extends as slender "sleeves" so as to reach the portal tracts and create portal-central (P-C) bridging, leaving zones 1 and 2 as a spheroid parenchymal clump. These were examined on serial histologic sections of the livers, which were subjected to 3 -D reconstruction with the aid of a computer system. The geometric properties of the interfaces between the surviving and necrotic areas were analyzed by $3-\mathrm{D}$ tangent counting technique of DeHoff modified for 3-D application. It was shown by reconstruction that necrosis was uniformly distributed around the hepatic venules. In acute cases, tangent counting showed that the surfaces of the surviving areas were mainly concave toward necrosis. In contrast, in chronic cases, the surfaces became mainly convex as a result of parenchymal regeneration. At no place, in acute or chronic cases, was necrosis shown to reach the portal tracts, and because of this, neither acini nor their agglomerates revealed themselves as a separate unit. All these findings contradict what one expects would be, so long as based on the assumption of the acinar model. ___ liver; zonal necrosis; threeD reconstruction; three-D tangent counting; acinar model (C) 1998 Tohoku University Medical Press
\end{abstract}

This paper is going to deal with the tenability of the acinar concept in the human liver. As well known, the acinus was proposed by Rappaport in the late 1950s as an anatomical unit of this organ (Rappaport et al. 1954, 1985; Rappaport

Received March 19, 1997; revision accepted for publication February 6, 1998.

Address for reprints: Yasuyuki Nakamura, M.D., Department of Pathology, Institute of Development, Aging and Cancer, Tohoku University, 4-1 Seiryomachi, Aoba-ku, Sendai 980-8575, Japan. 


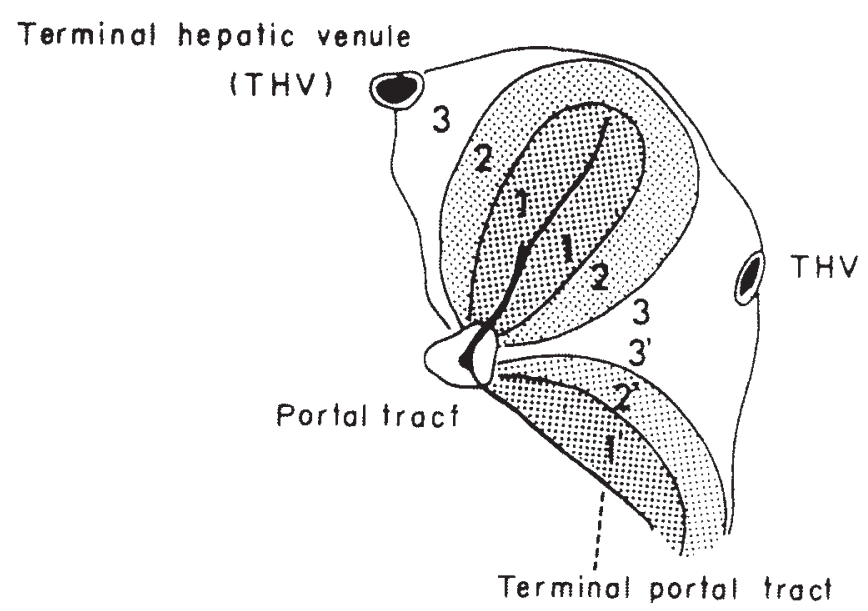

Fig. 1. A schema of Rappaport acinus, presenting zones 1 and 2 as forming spheroid parenchymal clumps, and zone 3 as wrapping an acinus as wedge-like "sleeves" reaching the preterminal portal tract.

1957). Since then, the model of Fig. 1 has growingly dominated those working in liver anatomy and histopathology. Rappaport asserts in this model that the acinus is definable as an independent unit, with zones 1, 2 and 3 concentrically arranged around a terminal portal venule. This zonation was given with the implication that the parenchymal tissue in zone 3 is the last to be irrigated by portal blood, where the hepatocytes must particularly be vulnerable to injury in acute ischemia or intoxication. One may agree that in such cases, the area around a terminal hepatic venule is the first to be destroyed. However, it has yet to be investigated whether necrosis occurs so as to separate an "acinus" from the neighboring ones as illustrated in the model. Consider what if, in actual cases, necrosis of zone 3 failed to extend in a "sleeve-like" fashion and reach the portal venule as given by the model. The clump of parenchyma which remains exempted from necrosis, i.e., the zones 1 and 2, must only partially be demarcated and remain fused with neighboring areas. This is far from a state in which a unit is definable. Whether or not the clump is really separable is closely related with its shape; according to the acinar model, the surviving parenchyma is separable because it has a surface convex toward the necrotic zone. However, as in the following, convexity does not appear to be a common finding in human livers with acute zonal necrosis where the very shape of acinus should have revealed itself (Fig. 2).

Thus, we have doubt on whether the acinar model really can explain the development of zonal pattern, at least in humans (Takahashi 1970). If it cannot, the ground upon which this model was designed should be subject to reexamination. The present study was undertaken to visualize the spatial distribution of zonal necrosis and its relation with the microvasculature of the liver by computer-aided 3-D reconstruction from serial sections. Cases were selected so as 

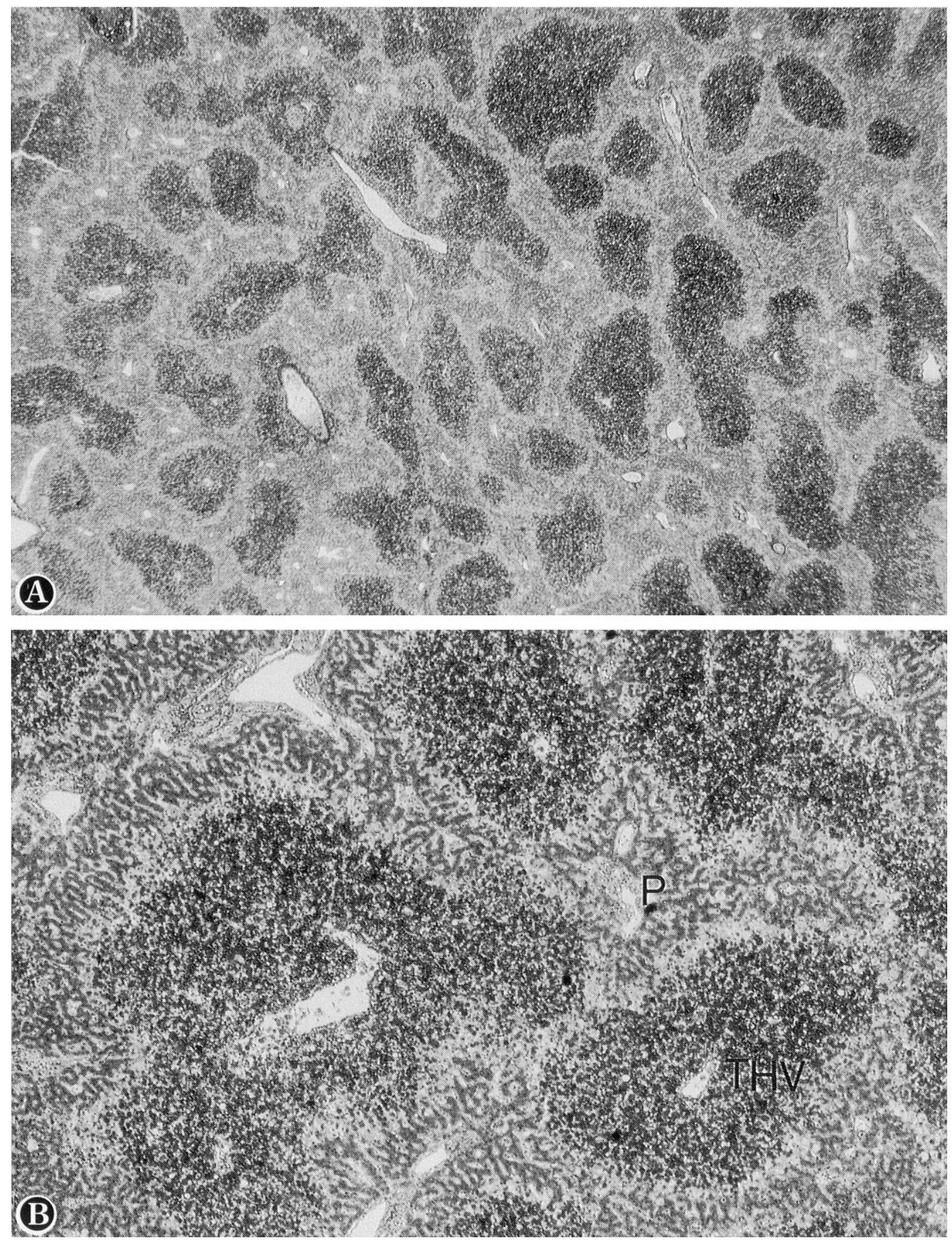

Fig. 2. The liver in Case 1. A) Areas of fresh coagulation necrosis are extending with characteristic zonal pattern. (Elastica-Goldner: original magnification $\times 4$ )

B) Necrosis is uniformly distributed around the terminal hepatic venules (THV). The borderlines between the necrotic and surviving areas mostly look convex toward the latter. P: portal tract. (Elastica-Goldner: original magnification $\times 10$ )

to include not only those with fresh zonal necrosis but also those where necrosis had been long-standing, since the shape of the lesion seemed to change more or less with the course of time: In an acute phase of intoxication (Fig. 2) necrosis appears 


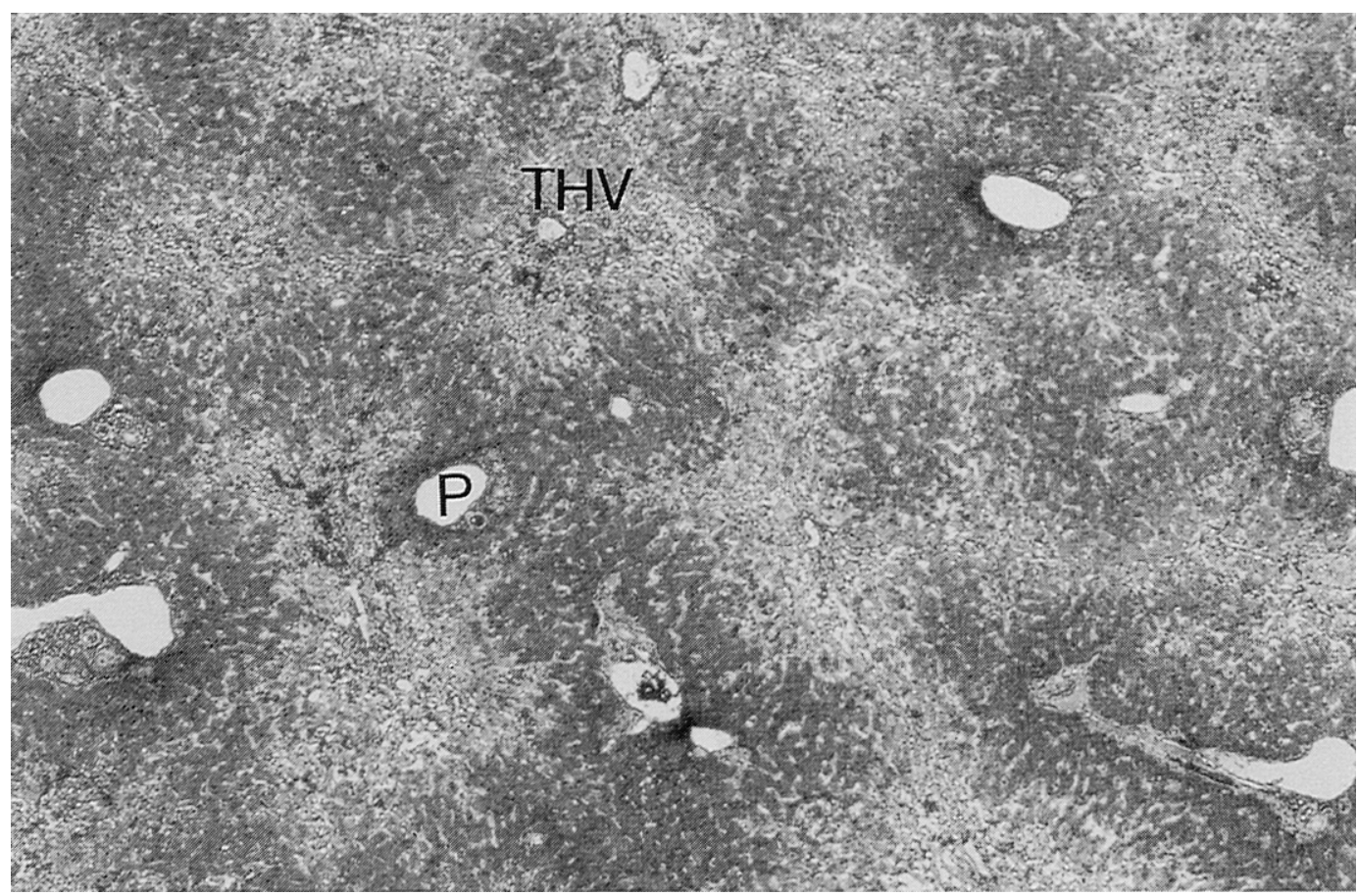

Fig. 3. The liver in Case 5. Necrosis is clearly demarcated and found around the peripheral hepatic veins. However, there are nodular contours in the remaining parenchyma, bound with curves convex toward necrosis, giving an impression different from acute necrosis. (Elastica-Goldner: original magnification $\times 4$ )

to have boundaries concave toward itself, while in the long-standing heart failure (Fig. 3), convex boundaries seem to prevail. However, this is essentially a matter of geometry in the space, which has to be studied from a 3-D structure point of view; whether the bounding interface is concave or convex can be determined by comparing the solid angle $\omega$ it creates. Thus, we modified the tangent counting technique originally developed for 2-D angles (DeHoff 1968) so that we may apply to the estimation of 3-D. As to be shown, this allowed us to describe whether and to what degree the interfaces between the necrotic and the surviving areas are concave or convex. Because we probably are going to show for the first time an application of this technique to pathology, its basic principles will also be introduced.

\section{Cases Examined}

Livers from five patients with zonal necrosis were selected from the autopsy material of Department of Pathology, Tohoku University Hospital and Saka General Hospital (Table 1). Two of the patients, Cases 1 and 2, died in an acute phase of cresol intoxication, in whom death occurred 60 and 90 hours after taking the poison, respectively, with suicidal intention. In both of the cases the liver was quite suitable for this study since there was necrosis of typically zonal pattern, clearly demarcated and distributed around the terminal hepatic venules.

In the other three patients, Cases 3,4 and 5, a chronic heart failure was 
TABLE 1. The examined cases

\begin{tabular}{|c|c|c|c|}
\hline \multicolumn{2}{|c|}{ Age and sex } & Clinical diagnosis & History \\
\hline \multicolumn{4}{|c|}{ Acute zonal necrosis } \\
\hline Case 1 & $23 \mathrm{~F}$ & Cresol intoxication & 60 hrs after ingestion of cresol \\
\hline Case 2 & $62 \mathrm{~F}$ & Cresol intoxication & $90 \mathrm{hrs}$ after ingestion of cresol \\
\hline \multicolumn{4}{|c|}{ Chronic zonal necrosis } \\
\hline Case 3 & $56 \mathrm{M}$ & Amyloidosis of heart & Cardiac failure for 2 years and 8 months \\
\hline Case 4 & $25 \mathrm{~F}$ & $\begin{array}{l}\text { Primary pulmonary } \\
\text { hypertension }\end{array}$ & Signs of cardiac failure for 10 months \\
\hline Case 5 & $46 \mathrm{M}$ & Aortic regurgitation & $\begin{array}{l}4 \text { years and } 3 \text { months after operation for } \\
\text { aortic regurgitation }\end{array}$ \\
\hline
\end{tabular}

mainly responsible for death which took place after an illness lasting 10 months or longer. Here again, the liver presented zonal necrosis around terminal hepatic venules, but there were signs of parenchymal regeneration producing more or less nodular pattern.

\section{Serial sectioning}

In all the five cases, autopsy was performed within 2 hours post mortem. A specimen about $3 \times 2 \mathrm{~cm}$ and $0.4 \mathrm{~cm}$ thick was taken from each of the livers, fixed in $10 \%$ formaldehyde solution and embedded in celloidin-paraffin. From each block, more than 250 serial microscopic sections were prepared at $4 \mu \mathrm{m}$ in thickness of a single section, and stained with Elastica-Goldner method (Goldner 1938).

\section{Computer-assisted 3-D reconstruction}

The serial sections were submitted to computer-assisted 3-D graphic reconstruction to visualize the distribution of necrosis and its relation with the liver vasculature. In the first of a set of serial sections, an area of liver tissue about $6 \times$ $4 \mathrm{~mm}$ was selected for reconstruction. Using a profile projector (model V-16C; Nikon, Tokyo), the area was projected onto a sheet of tracing paper at a magnification of $\times 50$, and the contours of all the portal and hepatic veins and venules contained in the area were faithfully delineated. Also, the borderlines of the necrotic and the surviving areas were drawn. This procedure was repeated sequentially every four sections, i.e., at a between-sections interval of $16 \mu \mathrm{m}$, until a tissue thickness of $800 \mu \mathrm{m}$ was covered with a sequence of fifty 2-D pictures. This was to contain a hepatic lobule whose dimension was estimated to be 700-800 $\mu \mathrm{m}$ in diameter (Takahashi et al. 1968). Then the serial pictures thus produced were placed on a digitizer one after another, and the profiles were inputted into a workstation (model 345SRX; Hewlett-Packard, Fort Collins, CO, USA) by tracing with a cursor. The system is a revised version of the one developed at this department upon a desktop computer (Yaegashi et al. 1987). On inputting, the 
contours of portal veins, hepatic veins and boundaries of necrotic areas were stored as separate files. When all the pictures have been inputted, the computer was ready to integrate a 3-D picture in the display. A picture from any desired angle can be produced, but we usually fixed our viewpoint so as to observe stacked 2-D sections from a vertical angle.

With this visualization of $3-\mathrm{D}$, we aim to make clear whether or not an "acinus" is a structure separable from each other. This implies to examine, among others, whether there really is such "sleeve-like" extension of necrosis from around the hepatic venules, enveloping an acinus as illustrated in Rappaport model.

The concavity-or-convexity of the parenchymal areas determined by 3-D tangent counting

The shape of zonal necrosis is closely related with the geometry of the interface between the surviving parenchyma and necrosis. This is a continuous surface which at some places appears concave toward the necrotic areas, but one may also find convexities somewhere. So we briefly describe the principle to determine whether convexity or concavity is predominant, and to what degree. This is to quantify the curvature created by the surfaces in a certain volume, with the solid angle $\omega$. The angle can easily be estimated if we apply the 3-D tangent counting method while performing graphic reconstruction.

The geometric properties of surfaces

Generally, there are three different types of surfaces: convex, concave and saddle (Fig. 4). In case of zonal necrosis let us take the surviving parenchyma as

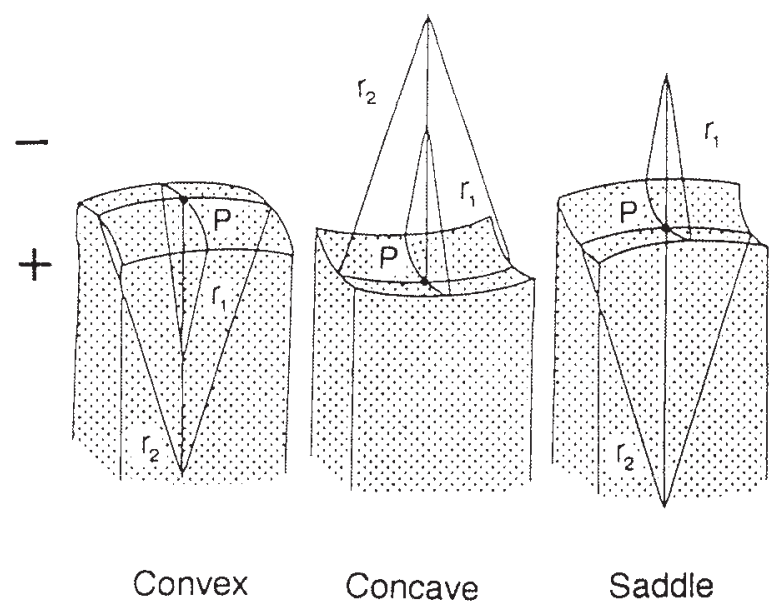

Fig. 4. Three types of interface between the surviving parenchyma (lower, shaded) and necrosis (upper, void): convex (left), concave (center) and saddle (right) surfaces. In a convex surface, the principal radii of curvature at $\mathrm{P}, \mathrm{r}_{1}$ and $r_{2}$, are defined as lengths toward the inside, while in a concave surface, both of the radii are in the outside. In a saddle, one of them comes inside, the other in the outside. 
the structure of interest enveloped by a continuous surfaces of varying types. Take, as an example, a point $P$ in a small patch of surface convex toward the outside (Fig. 4 left). At this point, a pair of principal radii of curvature $r_{1}$ and $r_{2}$, the maximum and the minimum radii, are defined as lengths toward the inside of parenchyma. The surface, when cut by a plane through $P$, appears on the section as a curve convex toward the outside. Conversely, at a concave surface (Fig. 4 center), both of the principal radii of curvature turn out to be in the outside, where cutting always generates a concave curve on section. However in the third type of surface, the saddle, the curve emerging on a section can be either convex or concave, depending on the direction of cutting (Fig. 4 right). Here, one of the principal radii of curvature comes to be inside, while the other is in the outside of the surviving parenchyma. Because the saddle parts occur very often in our material and in fact have the largest share in the total curvature, studies from a 3-D geometry point of view cannot be avoided.

\section{The solid angle}

We can define the curvature of the surface of surviving areas with the solid angle $\omega$, according to a rationale that follows. In the left part of Fig. 5, we consider a segment of 2-D arc, $L$ in length, of a circle $R$ in radius. The curvature of the arc is measured with the angle $\theta$ it "subtends" at the center of the circle. Thus

$$
\theta=L / r \text {. }
$$

Now we expand this relation into 3-D. In Fig. 5 right, suppose a sphere of $R$ in radius and a patch of surface on it with an area of $S$. This patch too, has a curvature corresponding to the solid angle $\omega$ it subtends at the center of the sphere. As in the case of $2-\mathrm{D}, \omega$ is given by

$$
\omega=S / r^{2} \text {. }
$$

For the whole sphere, $\omega=4 \pi$, since $S=4 \pi r^{2}$. Thus, the solid angle expresses the
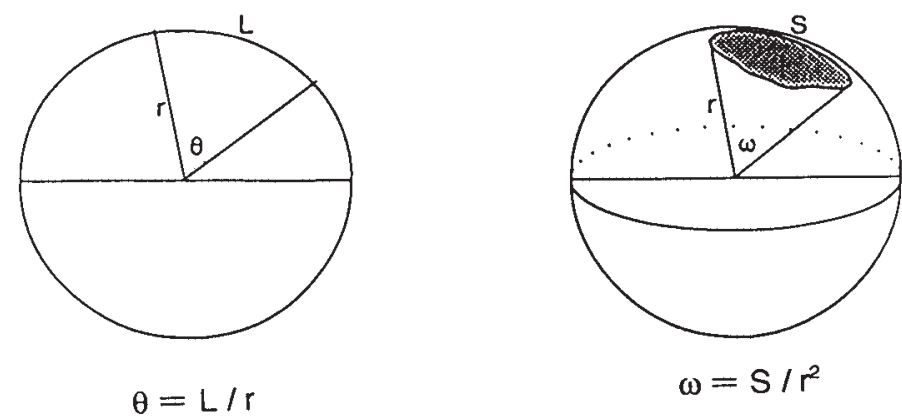

Fig. 5. The angles created by curvature. (Left) A 2-D arc of a circle $\mathrm{R}$ subtends an angle $\theta$ at the center of a circle. (Right) Similarly in the 3 dimensions, we can define the angle $\omega$, the solid angle, created by the curvature of a patch $\mathrm{S}$ of a sphere. 

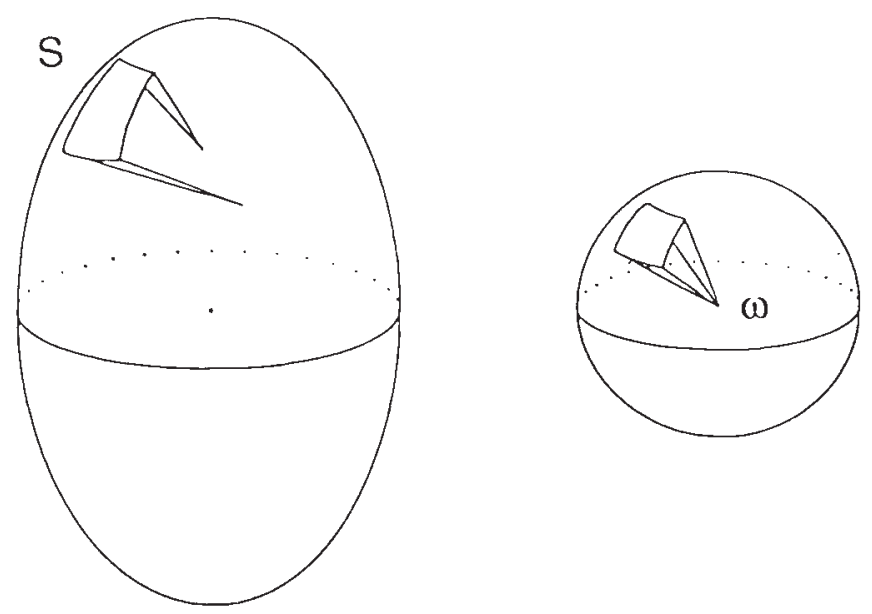

Fig. 6. The solid angle $\omega$ of a patch from an arbitrary surface $\mathrm{S}$ (left) can be defined by replacing the normals elected along the contour with unitary vectors of the same direction which then are translated onto a sphere (right).

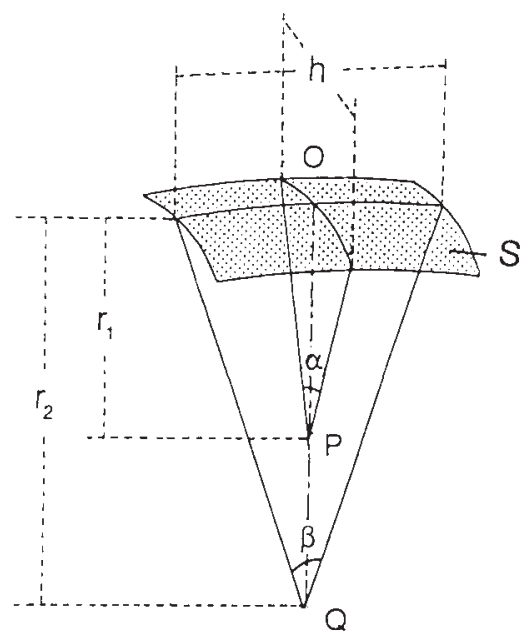

Fig. 7. Factors implicated in the definition of Gauss' curvature. Assume a square patch in the surface of parenchyma with edges of $h$ in length and with the minimum and maximum radii of curvature, $r_{1}$ and $r_{2}$, respectively. Then the solid angle w corresponding to $\mathrm{S}$ is given by

$$
\omega=\mathrm{h}^{2} \mathrm{~K}=h^{2} / r_{1} r_{2},
$$

where $\mathrm{K}$ is Gauss' curvature defined as

$$
\mathrm{K}=\mathrm{k}_{1} \mathrm{k}_{2}=1 / r_{1} r_{2} \text {. }
$$

spread of space from a point in the form of cone.

This can be generalized into any arbitrarily taken small patch of surface. As in Fig. 6 left, a patch from an arbitrary surface differs from that of sphere because the normals erected along the contour do not converge upon a point. Still, we can define $\omega$ by replacing the normals with unitary vectors of the same direction which then are converged on a point by parallel translation (Fig. 6 right). The 
tips of the vectors define a transformed surface, and consequently, $\omega$. Now assume, as in Fig. 7, a square patch in the surface of parenchyma with edges of $h$ in length, with the minimum and maximum radii of curvature, $r_{1}$ and $r_{2}$, respectively. Then the solid angle $\omega$ subtended by this patch can be written as

$$
\omega=h^{2} / r_{1} r_{2}
$$

according to principles of solid geometry. Here we discriminate between plus and minus rs; a radius is plus when it stretches toward the interior of parenchyma, and minus in the case of exterior radius as in Fig. 4. Thus, in both convex and concave surfaces $\omega$ is plus because $r_{1} r_{2}$ is $[++]$ in the former and $[--]$ in the latter. Conversely, a saddle surface is characterized by a minus $\omega$ with a combination of $[+-]$. The equation (1) can easily be derived if one attempts to describe w using Gauss' curvature.

Now let us examine in some examples what relation we have between the shape of 3 -D surface and $\omega$ it creates. In a sphere, $\omega=+4 \pi$, as shown above. This holds true not only for a sphere but for any convex bodies like an ellipsoid, where $\omega$ is constantly $+4 \pi$ for one body.

In the next place, imagine a shape of two spheres partially aggregated as in Fig. 8. Here, since both of the spherical parts are larger than a hemisphere, $\omega$ created by the convex part of each of them can be described as $[+2 \pi+\alpha]$ and $[+2 \pi+\beta]$, respectively. However, between the two spheres, there is a circumferential zone of saddle surface. This saddle is responsible for producing a minus angle of $-[\alpha+\beta]$. By adding this to the above, $\alpha$ and $\beta$ are offset, and we have a total net $\omega$ of $+4 \pi$ for this closed surface which is of rather complicated shape. Thus, a saddle emerges at a place of transition from convexity to concavity, at a constriction, or, as in the following, at the margin of a hole.

We now expand our consideration to a closed surface having "holes." Shown in Fig. 9 left is a torus having one hole. Along the inner surface of the hole there

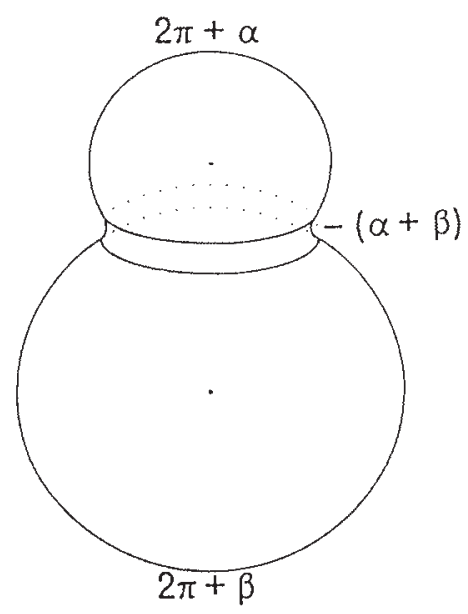

Fig. 8. Solid angle of structures more complex than a sphere. In a closed surface like this, the total net $\omega$ is always $+4 \pi$ if there is no hole. 

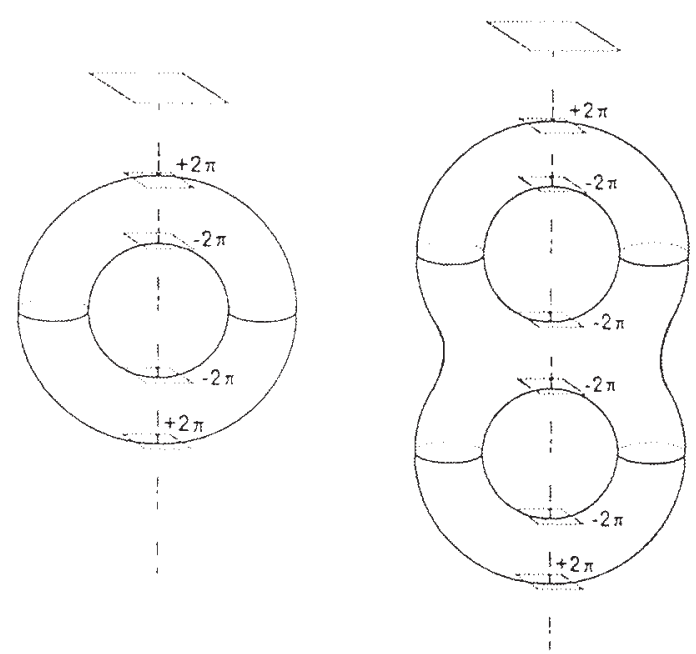

Fig. 9. Solid angle of a closed surface having "holes." In a torus having one hole (left), there is a belt of saddle surface along the inner surface of the hole, which generates a negative $\omega$ of $-4 \pi$, making the total solid angle zero. If a surface contains two holes as in the right, then the total solid angle is $-4 \pi$.

is a belt of saddle surface which generates a negative $\omega$ of $-4 \pi$. It offsets an $\omega$ of $+4 \pi$ which, without a hole, would have been generated by the closed surface. Thus, $\omega=0$ for a torus. If a surface contains two holes as in Fig. 9 right, then the net solid angle produced is

$$
+4 \pi+2(-4 \pi)=-4 \pi
$$

Generally, if we have $m$ closed surfaces in a space and there are $c$ holes, the total solid angle generated in the space is

$$
\omega(\text { net })=4 \pi(m-c),
$$

where $c$, the number of holes, corresponds to what in topology is called the genus. If a closed surface has a number of holes, it is a 3-D network as a whole. In this equation, one can see how closely the solid angle generated by surfaces correlates with the connecting relation of network into which they are assembled.

\section{Estimation of the solid angle by 3-D tangent counting}

The sum of solid angles contained in a volume, absolute or net, can quite easily be estimated if one applies the tangent counting method. This is a technique proposed by DeHoff (1968) for the estimation of total curvature generated by 2-D curves (DeHoff 1977), but here we introduce its 3-D modification.

Fig. 10 demonstrates a rectangular space containing surfaces of various shape. Imagine that we slowly move the ceiling of the space ABCD downward by parallel translation, a procedure we call "sweeping" of the space. Then it may be conceivable that this plane comes to touch with the surfaces at various points, at each of which it produces a tangent. It may be clear that statistically, a tangent occurs at every $+2 \pi$ solid angle at convexity as well as concavity, whereas at a 


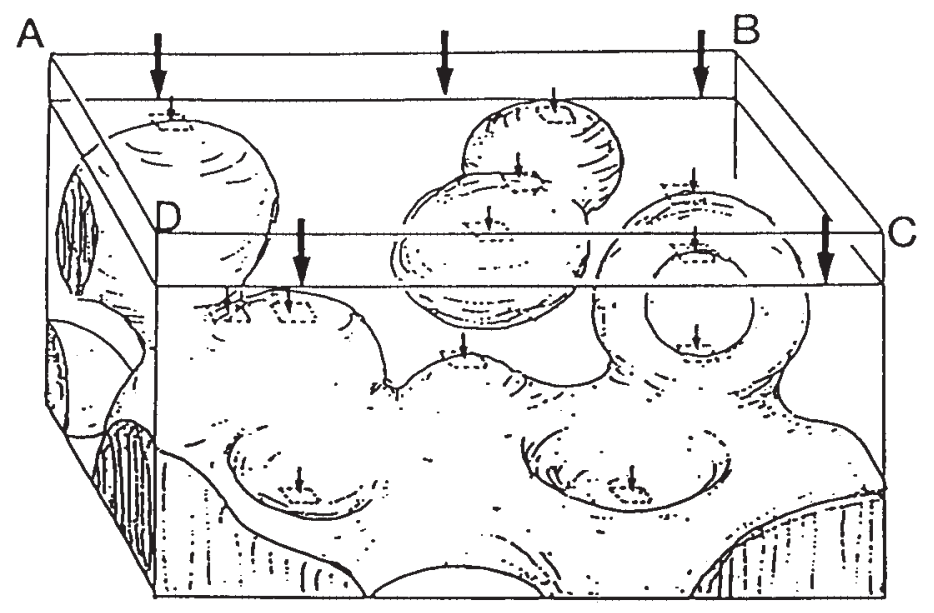

Fig. 10. Estimation of solid angle by 3-D tangent counting. Moving the ceiling $\mathrm{ABCD}$ of this space downward by parallel translation, several tangents occur at convex, concave or saddle portions of the surface.

saddle plane, at an angle of $-2 \pi$. Accordingly, if we count the number of tangents $n$ occurring at convex, concave and saddle separately, the total $\omega$ generated by each component is given by

$$
\begin{aligned}
& \omega(\mathrm{cvx})=+2 \pi n(\mathrm{cvx}) \\
& \omega(\mathrm{ccv})=+2 \pi n(\mathrm{ccv}) \\
& \omega(\mathrm{sdl})=-2 \pi n(\mathrm{sdl})
\end{aligned}
$$

where $n(\mathrm{cvx}), n(\mathrm{ccv})$ and $n(\mathrm{sdl})$ denote the number of tangents generated at convex, concave and saddle parts of interface. Thus the total net $\omega$ contained in the space amounts to

$$
\omega=2 \pi\{n(\mathrm{cvx})+n(\mathrm{ccv})-n(\mathrm{sdl})\} .
$$

The counting of tangents was performed upon serial sections (Fig. 11). By looking through a stack of sequential 2-D pictures in the display, points are easily found where a tangent emerges when the space is "swept" by a plane parallel to the sections. Also, there are no difficulties in discriminating among the tangents occurring at convexity, concavity and saddle.

\section{RESULTS}

Three-D morphology of zonal necrosis

Shown in Fig. 12 is a 3-D picture of the liver in an acute phase of cresol intoxication (Case 1), the microscopic appearance of which has already been shown in Fig. 2. It may be clear 3-dimensionally that the surface of the surviving parenchyma, presented with half-transparent yellowish zones, comprizes concave, convex and saddle parts but concavity is likely to be more common than convexity. At no place, necrosis was found extending as a sleeve-like flap 
reaching the portal tract. The parenchymal areas that survived necrosis were only uniformly surrounding segments of portal veins, without being separated into "acini" demarcated from each other. These are findings which may be least expectable from the acinar model. Occasionally there are connections between neighboring areas of necrosis, disclosing that the overall shape of necrosis is a loose 3 -D network. In the other case of cresol poisoning (Case 2), essentially the same results were obtained.

Of the three patients who died after a long-standing cardiac failure, the liver of Case 5 is to be shown. Although, here again, necrosis was distributed around the peripheral hepatic venules as in Fig. 3, there was a difference from the acute necrosis; sustained regeneration gave the parenchymal areas nodular contours with the boundaries convex toward necrosis. Here it may seem as if the acini of Rappaport revealed themselves as unitary structures.

However, this was disproved by 3-D reconstruction of Fig. 13. Certainly, regeneration changed the interface into a shape where convexity to necrosis appears to be strengthened. However, here too, "acini" were not separated. Necrosis, only wrapping the small hepatic venules, proved nowhere to be so extensive as to reach the portal tract.

\section{Geometric properties of the lesion revealed by 3-D tangent counting}

As above, the shape of interface between the parenchymal and necrotic zones appears to change with the duration of disease. This was quantified by estimating the solid angles created by the interface in a volume of tissue. Listed in Table 2 are the numbers of tangents, $n(\mathrm{cvx}), n(\mathrm{ccv})$ and $n(\mathrm{sdl})$, generated at a "sweeping" of the test space. The ratio of these numbers can directly be translated into the share of the corresponding solid angles $\omega(\mathrm{cvx}), \omega(\mathrm{ccv})$ and $\omega(\mathrm{sdl})$, because, as above, the value of $\omega$ is proportional to the tangent number. From the tangent numbers, $\omega($ net) was calculated according to (3), which was then

TABLE 2. Geometric properties of the lesion revealed by 3-D tangent counting

\begin{tabular}{|c|c|c|c|c|c|c|c|}
\hline & \multirow{2}{*}{$\begin{array}{c}\text { Weight } \\
\text { of liver }(\mathrm{g})\end{array}$} & \multirow{2}{*}{$\begin{array}{c}\text { Sampling } \\
\text { volume }\left(\mathrm{cm}^{3}\right)\end{array}$} & \multicolumn{3}{|c|}{ The number of tangents } & \multirow{2}{*}{$\begin{array}{l}\omega(\text { net })_{0} \\
\text { in } \mathrm{cm}^{3}\end{array}$} & \multirow{2}{*}{ Genus } \\
\hline & & & $\mathrm{n}(\mathrm{ccv})$ & $\mathrm{n}(\mathrm{cvx})$ & $\mathrm{n}(\mathrm{sdl})$ & & \\
\hline \multicolumn{8}{|l|}{ Acute } \\
\hline Case 1 & 1180 & 31.0 & $59(25 \%)$ & $14(6 \%)$ & $169(69 \%)$ & $-192 \pi$ & $1.83 \times 10^{6}$ \\
\hline Case 2 & 990 & 17.5 & $26(22 \%)$ & $4(3 \%)$ & $91(75 \%)$ & $-122 \pi$ & $1.73 \times 10^{6}$ \\
\hline \multicolumn{8}{|l|}{ Chronic } \\
\hline Case 3 & 830 & 25.6 & $16(4 \%)$ & $89(24 \%)$ & $222(70 \%)$ & $-234 \pi$ & $1.90 \times 10^{6}$ \\
\hline Case 4 & 1140 & 29.2 & $23(4 \%)$ & $158(27 \%)$ & $415(70 \%)$ & $-468 \pi$ & $4.57 \times 10^{6}$ \\
\hline Case 5 & 946 & 21.7 & $23(4 \%)$ & $136(22 \%)$ & $467(75 \%)$ & $-616 \pi$ & $6.71 \times 10^{6}$ \\
\hline
\end{tabular}

This table presents the numbers of tangents, $n(\mathrm{cvx}), n(\mathrm{ccv})$ and $n(\mathrm{sdl})$, generated at a "sweeping" of the test space. Consequently, we can determine the topological properties of the lesion by estimating the genus. 
divided by the volume of the test space to obtain $\omega$ (net) $)_{0}$, the net angle per a unit volume $\left(1 \mathrm{~cm}^{3}\right)$. Further, the total net angle $\omega(\text { net })_{\mathrm{T}}$ in the whole organ was estimated by

$$
\omega(\text { net })_{\mathrm{T}}=\omega(\text { net })_{\mathrm{O}} \times\{(\text { liver weight in } \mathrm{g}) / 1.0\}
$$

assuming that the specific gravity of the liver is 1.0 .

In all the five livers, $\omega(\mathrm{sdl})$, the angle produced by saddle parts of interface, was the largest, occupying about $70 \%$ of the total solid angle, thus making $\omega(\text { net })_{\mathrm{T}}$ a large minus value. As shown in (2), this implies that the interface has many holes, since a liver is considered to have a totally united interface and therefore, $m=1$. In other words, the overall geometry of the parenchymal as well as the necrotic areas is a finely meshed 3-D network. The difference between the acute (Cases 1 and 2) and chronic zonal necrosis (Cases 3, 4 and 5) is clear. In the former, the shape of the angles created by concave parts was $25 \%$ and $22 \%$, respectively, while that by convex parts was only $6 \%$ and $3 \%$. In contrast, in the latter cases, the angle created at concavity was only about $4 \%$ whereas that at convexity was as much as $24 \%, 27 \%$ and $22 \%$. These may be what one expects from the impression given by the $3-\mathrm{D}$ reconstruction pictures. Thus, the shape of zonal necrosis changes with the advancement of parenchymal regeneration that intervenes with the course of time and complicates the picture.

\section{Topological properties of zonal necrosis}

As shown above, the geometry of the interface is a 3-D network. How densely the network is knit is given by the number of holes, or meshes it contains. This is given by the value of $\mathrm{c}$ in (2), which corresponds to the genus in terms of topology. By giving $\omega(\text { net) })_{\mathrm{T}}$ to $\omega$ (net) in (2) and neglecting $m$ (which is 1 ), a rough estimate of the genus was obtained. As in Table 2, the genus proved to be within a comparatively narrow range of 1.7 to $1.9 \times 10^{6}$ in both of the cresol cases. In the three cases with chronic heart failure, it was in about the same range in one of the cases (Case 3), while in the other two cases it was larger, reaching $6.71 \times 10^{6}$ in Case 5 where the disease lasted for the longest duration.

\section{Discussion}

The change of liver due to acute ischemia or intoxication is experienced very often at autopsy. Usually it presents as zonal necrosis of "centrilobular" type distributed around small hepatic veins and venules. From a microanatomy point of view, this may be the very state in which the basic unitary structure of the liver is expected to reveal itself. Particularly the acinus of Rappaport (Rappaport et al. 1954, 1985; Rappaport 1957) is likely to be visualized, since the acinar concept was designed upon what distribution he assumed the zonal necrosis would take in the liver. Recently we experienced livers from two patients dying in an acute phase of cresol intoxication, and those from another three, in whom death occurred 
after a long-standing cardiac failure. All the livers presented typical zonal necrosis while in the chronic cases, there was a modification of pattern resulting from intervening parenchymal regeneration. In these livers we studied the 3-D geometry of necrosis, with the aim to examine whether the zonal necrosis really takes such a shape as predicted by the acinar model.

The schema of acinus as Fig. 1 is well known. Here the acinus is defined as a parenchymal clump which has a terminal portal twig at the axis and comprises zones 1, 2 and 3 concentrically arranged from the axis to the surface. This was said to be the very order in which the portal blood would irrigate an acinus. Thus the zone 3 , the last to be irrigated, was regarded as being the most handicapped zone where the hepatocytes would particularly be liable to damage when the liver was subjected to severe ischemia or intoxication. On such occasion, the shape of acini must clearly be visualized, with zone 3 uniformly destroyed, standing in sharp contrast against zones 1 and 2 where hepatocytes remain alive. Particularly worth attention is the shape of zone 3 which was drawn so as to be extending toward the portal tract as a slender wedge-like "sleeve" and reach there. Consequently, zone 3 necrosis, if really occurs, would give rise to the formation of bridging, a "portal-central (P-C)" bridging according to the wording commonly used in liver histopathology. On the other hand, the area corresponding to zones 1 and 2 would be exempted from necrosis and remain as a spheroid clump, with a surface convex toward the necrotic zone. Rappaport (1976) classified zonal necrosis into perivenular, triangular and other subtypes, and here too, the surviving parenchyma was illustrated as having a convex surface. It seems likely that both the P-C bridging by zone 3 and the convexity of the surviving zones 1 and 2 were assumptions necessary for defining "acinus" as an independent anatomical unit. These must have been assumed because otherwise, zones 1 and 2 of the neighboring "acini" would be fused, making the individual acini inseparable. If the surface were concave instead of being convex, apparently there would be no room for zone 3 to make a "sleeve."

The present study was undertaken to examine whether in the human liver, the necrotic and surviving zones really take such a shape. We resorted to two methods. One is computer-assisted 3-D reconstruction which was used to examine whether in the livers with acute necrosis, there is such "sleeve-like" extension of necrosis that may separate individual acini. The other is 3-D tangent counting which we applied to the interface delimiting the surviving parenchyma from necrotic areas. This allowed to define the geometric properties of the interface with the solid angle $\omega$ created by its curvature in a test volume. By comparing between $\omega(\mathrm{ccv})$ and $\omega(\mathrm{cvx})$, i.e., the solid angle created by concavity and that by convexity, one can estimate which of these dominates, and to what degree. The tangent counting is a simple technique of morphometry devised by DeHoff (1968, 1977) to define angles created by curves in a 2-D picture, but we attempted to apply the principle to the curvature of interface in $3-\mathrm{D}$ space. Since we seem to 
be presenting the first application of this technique to pathology, a brief geometric rationale was given.

Cresol (2-methoxy-4-methylphenol) is a disinfectant commonly used as a $50 \%$ cresol soap solution. In most clinical cases of cresol intoxication (Michael 1975; Bruce et al. 1976; Arthus et al. 1977; Haddad et al. 1979; Wiseman et al. 1980; Côtè et al. 1984; Jonsson and Voigt 1984), the poison was taken with suicidal intention. So far, there have been many autopsy reports of intoxication, but attention has been focussed on the corrosive changes of the upper digestive and respiratory tracts and skin (Michael 1975; Bruce et al. 1976; Arthurs et al. 1977; Wiseman et al. 1980; Jonsson and Voigt 1984), Heinz body anemia (Côtè et al. 1984), or acute tubular necrosis of the kidney (Michael 1975; Bruce et al. 1976). Liver changes have little been documented in humans, although experimentally, hepatocellular necrosis by cresol has well been established in rodents (Hattula et al. 1979; Thompson et al. 1994). In both of our cresol cases, the liver presented essentially the same picture; characteristic zonal necrosis around the hepatic venules. This finding, not having been reported in the previous reports, may itself be worth attention from a toxicological point of view. However, the livers, showing clearly demarcated necrosis, served as a material adequate for studying the geometry of lesion.

Our analyses betrayed in every respect what should be expected to result from the acinar model. The necrotic areas which developed in acute cresol intoxication, though presenting a typically zonal pattern, proved to be simply surrounding the hepatic venules. Nowhere were they found extending toward a terminal portal tract in "sleeve-like" fashion, much less reaching there. This implies that no P-C bridging arose. In the surface of the surviving parenchyma, concavity toward necrosis was much more predominant than convexity, although saddle planes accounted for the greatest part of the total $\omega$. Clearly, this is a situation in which no such spheroid parenchymal clump as illustrated in the acinar model can exist. From every viewpoint, the real morphology of acute zonal necrosis differs from Rappaport's model. Figs. 14 and 15 summarize what actually occurs and in what respect it differs from the model.

There are other agents which can induce acute zonal necrosis, e.g. ischemia, and we have extended our observation to such cases; here the livers were not subjected to 3-D analysis but were studied only on 2-D sectional level. However, no difference from the cresol livers was found in the pattern of lesion in any of them. We think therefore that the conclusion we have drawn above from the cresol livers may be generalized to acute zonal necrosis due to other causes, so long as the lesion is distributed around the hepatic venules. Possibly, the pattern common to these conditions may coincide with the distribution of pericentral enzymes described by Lamers et al. (1989). However we discuss about this later.

Why we obtained results unpredictable from the acinar model may be explainable from the following reasons. First, 3-D morphology of a lesion can 


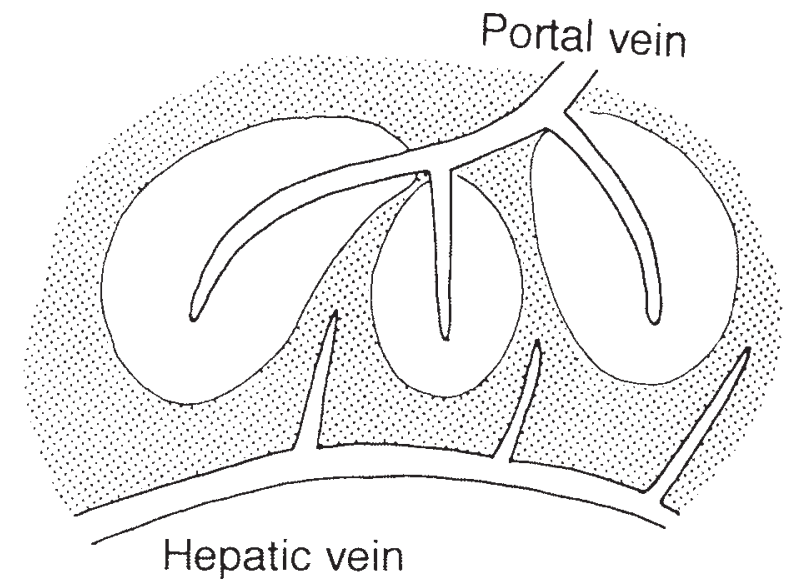

Fig. 14. The pattern of acute zonal necrosis expected from the acinar model. The shaded areas around the hepatic venules correspond to necrosis. If necrosis occurs in zone 3 , the surviving parenchyma (zones 1 and 2 ) must be left as a spheroid clump.

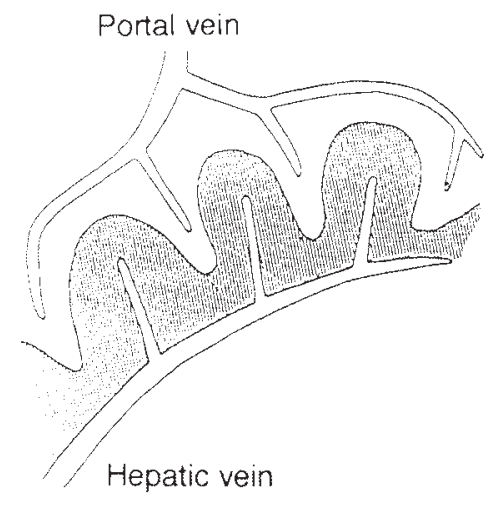

B

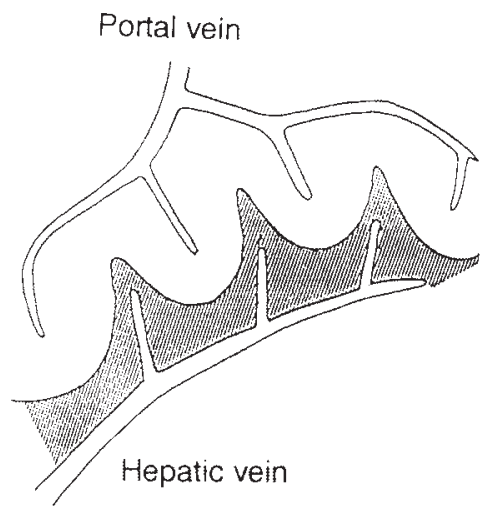

Fig. 15. The actual pattern of zonal necrosis (shaded areas).

A: The pattern of zonal necrosis above confirmed in acute cresol intoxication. The boundaries between the surviving and necrotic areas are mainly concave toward the latter. B: The pattern of zonal necrosis in livers with longstanding heart failure. The surfaces of the surviving areas are mainly convex toward necrosis. But necrosis never reaches the portal tracts.

often be different from what it seems in a 2-D section. The shape of zonal necrosis and its relation with the microvasculature of the liver are problems that cannot be solved without performing studies on 3-D level, while so far, the matter has been discussed only on 2-D sectional pictures. Second, it seems possible that the vasculature of the liver in humans differs more or less from other species, for instance that of rodents where the acinar concept was grounded.

In patients with chronic heart failure, zonal necrosis is sustained due to recurrent or long-standing ischemia. In such chronic cases, the lesion was shown to be modified: the surface of the surviving parenchyma turned to convexity from 
concavity of acute necrosis, as evidenced by the result of tangent counting where $\omega(\mathrm{cvx})$ is now predominant over $\omega(\mathrm{ccv})$. Clearly, this change is brought about by regeneration of hepatocytes which makes the shape of parenchymal areas more or less rounded. In a 2-D microscopic section, such areas can emerge as if they were cut from acini separated by zone 3 necrosis. However, 3-D reconstruction disclosed that this is more apparent than real. At no place, was necrosis confirmed to extend as "sleeves" toward the portal tracts and reach there. The surviving parenchyma, though having a surface mostly convex toward necrosis, was found only wrapping the portal venules as a continuous mantle.

The acinus was defined as a structural unit on the assumption that it may become visible when there is necrosis of what was expressed as zone 3. Accordingly, the zonal necrosis must be the very lesion in which the acinar concept should be the most helpful in explaining the pathogenesis. However, in our results, neither the acini nor their agglomerates revealed themselves even in typical cases of zonal necrosis. This contradicts what the acinar theory attempted to explain. Moreover, application of tangent counting revealed that in each of the livers with zonal necrosis, the surviving parenchyma as well as the necrotic zones are forming a fine network, with the total number of meshes in the organ exceeding $2 \times 10^{6}$. In other words, the surviving parenchyma is united into a large continuous network instead of being separated into solitary units. In every respect, we think the tenability of the acinar theory is doubtful.

Still, a problem remains. If the acinar concept does not help understand the morphogenesis of liver diseases, what an alternative should be introduced? Lamers et al. (1989) proposed a modification of Rappaport's concept based on observation that the pattern of acute zonal necrosis coincides with the distribution of some pericentral enzymes. We evaluate their contribution in that they disclosed an aspect of liver structure where the pattern of liver disease is decided by the distribution of enzyme. However, the accurate geometry of necrosis was left unexamined even in their modified schema. We are thinking of proposing another principle for the basic microstructure of the human liver. However, this will be introduced on another opportunity.

\section{References}

1) Arthurs, G.J., Wise, C.C. \& Coles, G.A. (1977) Poisoning by cresol. Anesthesia, 32, 642-643.

2) Bruce, A.M., Smith, H. \& Watson, A.A. (1976) Cresol poisoning. Med. Sci. Law, 16, 171-176.

3) Côtè M.A., Lyonnaris, J. \& Leblond, P.F. (1984) Acute Heinz-body anemia due to severe cresol poisoning: Successful treatment with erythrocytapheresis. Can. Med. Assoc. J., 130, 1319-1322.

4) DeHoff, R.T. (1968) Curvature and the topological properties of interconnected phases. In: Quantitative Microscopy, edited by R.T. DeHoff \& F.N. Rhines, McGrawHill, New York, pp. 291-325.

5) DeHoff, R.T. (1977) Stereological uses of the area tangent count. Geometrical 
probability and biological structures. Buffon's 200th Anniversary, 23, 99-113.

6) Goldner, J. (1938) A modification of the Masson trichrome technique of the routine laboratory purpose. Am. J. Pathol., 14, 237-243.

7) Haddad, L.M., Dimond, K.A. \& Schweistris, J.E. (1979) Phenol poisoning. Jacep, 8, 267-269.

8) Hattula, L.M., Reunanen, H. \& Arstila, A.U. (1979) Toxicity of 4-chloro-O-cresol to rat: 1. Light microscopy and chemical observations. Bull. Environ. Contam. Toxicol., 21, $492-497$.

9) Jonsson, J. \& Voigt, G.E. (1984) Homicidal intoxication by lye- and parachlorcresol-cresol containing disinfectants. Am. J. Forensic Med. Pathol., 5, 5763.

10) Lamers, W.H., Hilberts, A., Furt, E., Smith, J., Jonges, G.N., van Noorden, C.J.F., Janzen, J.W.G., Charles, R. \& Moorman, A.F.M. (1989) Hepatic enzyme zonation: A reevaluation of the concept of the liver acinus. Hepatology, 10, 7276.

11) Michael, A.G. (1975) A household remedy misused. Fatal cresol poisoning following cutaneous absorption (A case report). Med. Sci. Law, 15, 65-66.

12) Rappaport, A.M., Borowy, Z.J., Lougreed, W.M. \& Lotto, W.N. (1954) Subdivision of hexagonal liver lobules into a structural and functional unit. Anat. Rec., 119, 11 33.

13) Rappaport, A.M. (1957) The structural and functional unit in the human liver (liver acinus). Anat. Rec., 130, 673690.

14) Rappaport, A.M. (1976) The microcirculatory acinar concept of normal and pathological hepatic structure. Beitr. Path. Bd., 157, 215-243.

15) Rappaport, A.M., MacPhee, P.J., Fisher, M.M. \& Phillips, M.J. (1985) The scarring of the liver acini (Cirrhosis). Virchows Arch. A., 402, $107-137$.

16) Takahashi, T., Orii, T. \& Kaneda, M. (1968) Precancerous condition of the human liver. Tohoku J. Exp. Med., 94, 203-224.

17) Takahashi, T. (1970) Lobular structure of the human liver from the viewpoint of hepatic vascular architecture. Tohoku J. Exp. Med., 101, 119-140.

18) Thompson, D.C., Perera, K., Fisher, R. \& Brendel, K. (1994) Cresol isomers: Comparison of toxic potency in rat liver slices. Toxicol. Appl. Pharmacol., 125, 5158.

19) Wiseman, H.M., Turner, W.M.L. \& Volans, G.N. (1980) Acute poisoning due to Wright's vaporizing fluid. Postgrad. Med. J., 56, 166 - 168.

20) Yaegashi, H., Takahashi, T. \& Kawasaki, M. (1987) Microcomputer-aided reconstruction. A system designed for studies in histology and histopathology. J. Microsc., 146,5565 . 


\section{Illustrations follow}


Fig. 11. The counting of tangents while performing 3-D reconstruction in a computer display. By looking through a stack of sequential 2-D pictures, one can easily find the tangents at convex, concave or saddle surfaces. In this 3-D reconstruction of the liver (Case 3), the hepatic veins are painted in blue and portal veins in pink. The boundaries between the surviving and necrotic areas are shown with yellow wireframes. One may recognize that the surface is convex at point $\mathrm{A}$ and saddle at point $\mathrm{B}$.

Fig. 12. Three-D reconstruction of the liver in an acute phase of cresol intoxication (Case 1). The hepatic veins are painted in light blue, portal veins in pink. The surviving parenchymal areas are shown as yellowish zones and the necrotic areas as black. Note that the surviving areas are uniformly distributed around the portal veins and the interfacial borders are mainly concave toward the necrotic areas.

Fig. 13. Three-D reconstruction of the liver of a patient who died after a longstanding cardiac failure (Case 5). In contrast to Fig. 12, the surviving parenchyma, shown as yellowish zones, have surfaces convex toward the necrosis. However, here again, the necrosis, shown in black, never reaches the portal veins. 

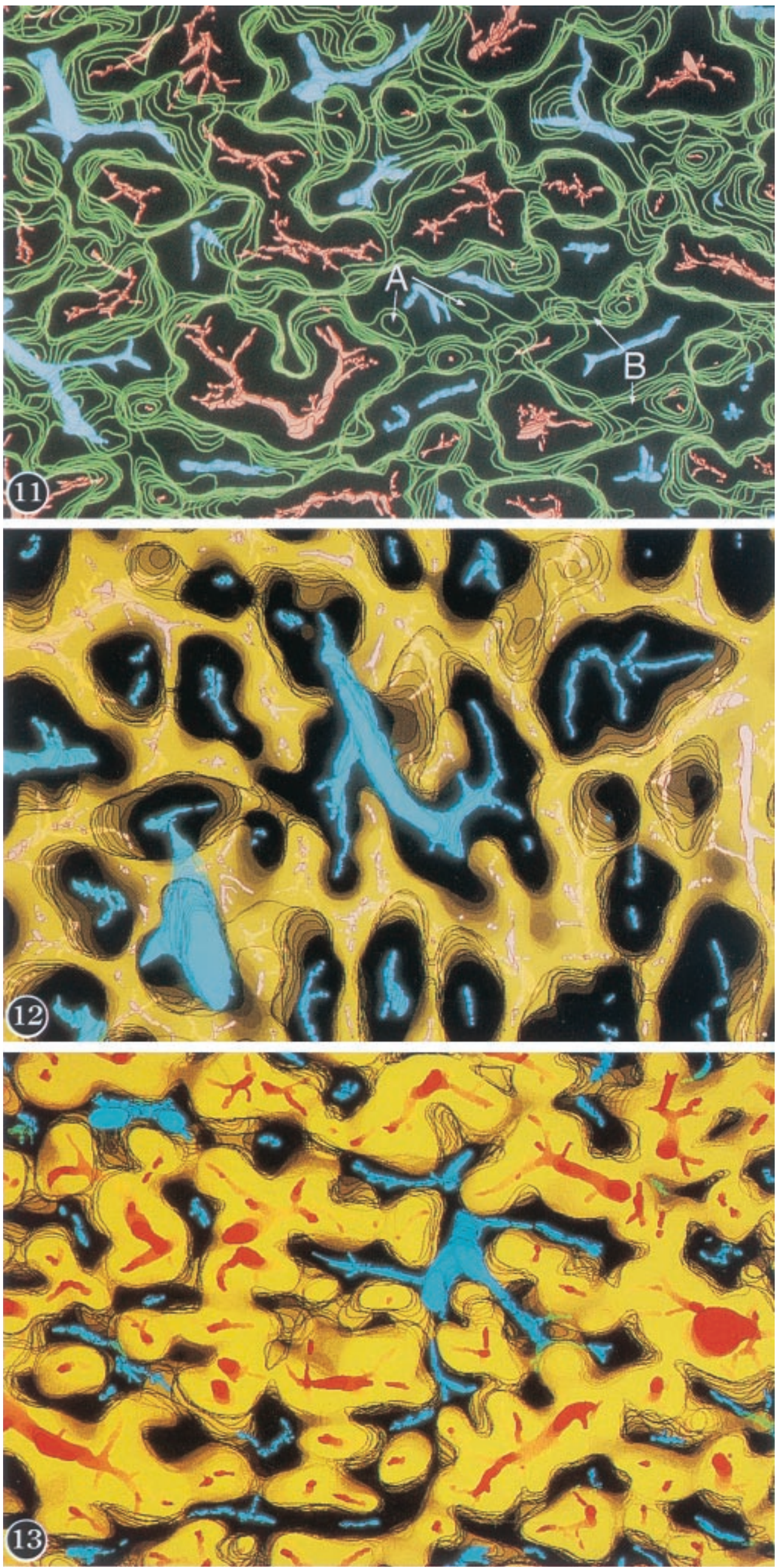